\title{
EHF suppresses cancer progression by inhibiting ETS1-mediated ZEB expression
}

\author{
Kaname Sakamoto ${ }^{1,2}$, Kaori Endo ${ }^{1,3}$, Kei Sakamoto ${ }^{4}$, Kou Kayamori ${ }^{4}$, Shogo Ehata $\mathbb{0}^{5}$, Jiro Ichikawa $\mathbb{1 0}^{6}$, Takashi Ando ${ }^{6}$, \\ Ryosuke Nakamura ${ }^{3}$, Yujiro Kimura ${ }^{3,7}$, Kunio Yoshizawa $^{7}$, Keisuke Masuyama ${ }^{2}$, Tomoyuki Kawataki ${ }^{8}$, Kunio Miyake ${ }^{9}$,

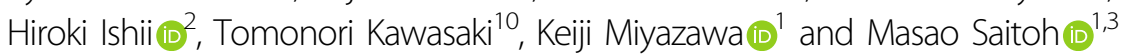

\begin{abstract}
ETS homologous factor (EHF) belongs to the epithelium-specific subfamily of the E26 transformation-specific (ETS) transcription factor family. Currently, little is known about EHF's function in cancer. We previously reported that ETS1 induces expression of the ZEB family proteins ZEB1/8EF1 and ZEB2/SIP1, which are key regulators of the epithelial-mesenchymal transition (EMT), by activating the ZEB1 promoters. We have found that EHF gene produces two transcript variants, namely a long form variant that includes exon 1 (EHF-LF) and a short form variant that excludes exon 1 (EHF-SF). Only EHF-SF abrogates ETS1-mediated activation of the ZEB1 promoter by promoting degradation of ETS1 proteins, thereby inhibiting the EMT phenotypes of cancer cells. Most importantly, we identified a novel point mutation within the conserved ETS domain of EHF, and found that EHF mutations abolish its original function while causing the EHF protein to act as a potential dominant negative, thereby enhancing metastasis in vivo. Therefore, we suggest that EHF acts as an anti-EMT factor by inhibiting the expression of ZEBs, and that EHF mutations exacerbate cancer progression.
\end{abstract}

\section{Introduction}

Squamous cell carcinoma is the predominant tumor type in head and neck cancer. Approximately two-thirds of patients with head and neck squamous cell carcinoma (HNSCC) present local metastases in bones and regional lymph nodes during their first visit to a hospital, and are therefore diagnosed as advanced stages ${ }^{1}$. Within 2 years after various treatments, including surgery, chemotherapy, and radiotherapy, more than $50 \%$ of patients have local recurrence or distant metastasis, resulting in extremely poor prognosis and overall survival ${ }^{1,2}$.

The process of cancer cell invasion and metastasis requires the loss of cell-cell interactions combined with the acquisition of motility, occasionally undergoing

\footnotetext{
Correspondence: Masao Saitoh (msaitoh-ind@umin.ac.jp)

'Department of Biochemistry, Graduate School of Medicine, University of Yamanashi, Yamanashi, Japan

2Department of Otolaryngology, Head and Neck Surgery, Graduate School of Medicine, University of Yamanashi, Yamanashi, Japan
}

Full list of author information is available at the end of the article epithelial-mesenchymal transition $(\mathrm{EMT})^{3-5}$. These phenotypic changes are regulated by extracellular matrix components, exosomes, and soluble factors, which regulate several EMT transcription factors (EMT-TFs), including the ZEB family of two-handed zinc-finger factors (ZEB1 [Zinc-finger E-box binding homeobox 1$] / \delta E F 1[\delta$ crystallin/E2-box factor 1] and ZEB2/SIP1 [Smad-interacting protein1]). Since EMT-TF gene mutations are rarely found in various cancers, EMT is thought to be a transient state, suggesting that cancer cells undergo a reversion process upon arriving at distant metastasized tissues called mesenchymal-epithelial transition $(\mathrm{MET})^{6,7}$. ZEB1/2 (ZEB1 and ZEB2) protein levels, in particular, correlate positively with EMT phenotypes and aggressiveness of breast cancer cell lines ${ }^{8}$. We have previously reported that ETS1, one member of the E26 transformation-specific (ETS) family of transcription factors ${ }^{9}$, induces ZEB expression and activates the $Z E B 1$ promoter in breast cancer cells ${ }^{10}$. We have also reported that ETS1 silencing represses expression of ZEB1/2 and partially restores their

\section{(c) The Author(s) 2021}

(c) (i) Open Access This article is licensed under a Creative Commons Attribution 4.0 International License, which permits use, sharing, adaptation, distribution and reproduction cc) in any medium or format, as long as you give appropriate credit to the original author(s) and the source, provide a link to the Creative Commons license, and indicate if changes were made. The images or other third party material in this article are included in the article's Creative Commons license, unless indicated otherwise in a credit line to the material. If material is not included in the article's Creative Commons license and your intended use is not permitted by statutory regulation or exceeds the permitted use, you will need to obtain permission directly from the copyright holder. To view a copy of this license, visit http://creativecommons.org/licenses/by/4.0/. 
epithelial phenotypes as well as their sensitivity to antitumor drugs.

The epithelium-specific ETS (ESE) transcription factors are a subgroup of ETS transcription factors defined by shared homology of the ETS domain, and include ELF3 (a. k.a. ESE1), ELF5 (a.k.a. ESE2), and EHF (a.k.a. ESE3) ${ }^{11}$. ELF3 is expressed in many different organs, whereas ELF5 and EHF expressions are restricted to glandular organs, including the salivary gland, mammary gland, and prostate gland. ESEs play crucial roles in normal development and have also been implicated in the pathogenesis of a wide range of cancers, playing both oncogenic and antioncogenic roles. In particular, ELF3 gene amplification occurs in various kinds of cancers, and ELF3 mutations frequently found in ampullary adenocarcinomas are heterozygous, suggesting that ELF3 may act as a haploinsufficient tumor suppressor ${ }^{12}$. However, the underlying mechanism of ELF3-mediated tumor suppression has not been elucidated. Recently, we found that ELF3 is highly expressed in the luminal subtype of breast cancer cells, and represses upregulation of ZEB1/2 by ETS1 in such cells ${ }^{10}$. Compared to ELF3, mutations in $E H F$ are relatively rare in human cancers and are not frequently deposited in public datasets. Occasional mutation and amplification of EHF occurs in a subset of cancers, such as ovarian, stomach, and bladder cancer. However, little is known about the roles of EHF in cancer ${ }^{11}$.

In this study, we examined the roles of EHF in HNSCC cells. We found that EHF is expressed at low levels in mesenchymal-like HNSCC cells, whereas ZEB1/2 expression levels are extremely high compared to other epithelial-like HNSCC cells. The EHF gene encodes two transcript variants, a long form (EHF-LF) and a short form (EHF-SF) variant, which are produced independently by either inclusion or exclusion of the first exon. EHF-LF is deposited as the longest form or precursor form in the NCBI database. Surprisingly, EHF-SF is localized to nuclei, and could inhibit ETS1-induced activation of the $Z E B 1$ promoter by promoting degradation of ETS1 proteins, whereas EHF-LF is localized to the cytoplasm or perinuclei, and failed to inhibit ETS1-induced activation, due to difference in subcellular localization. Most importantly, an EHF point mutation identified in cancer cells caused a loss of the capacity to inhibit ETS1induced phenomena, including upregulation of the ZEB1/ 2 and EMT phenotypes. We therefore propose that $E H F$ mutation was first discovered to promote cancer aggressiveness by promoting EMT.

\section{Results \\ EMT phenotypes in HNSCC cell lines}

We previously reported that ZEB1/2 (ZEB1 and ZEB2) expression is positively correlated with the EMT phenotypes of breast cancer cell lines ${ }^{8,13,14}$. To determine the EMT phenotypes of HNSCC cells, we investigated 11 HNSCC cell lines by immunoblot (IB) analysis and included two subtypes of breast cancer cell lines as positive and negative controls. MDA-MB-231 cells are categorized into the basal-like subtype of breast cancer with high levels of ZEB1/2 expression and low levels of Ecadherin expression, whereas MCF7 cells are categorized into the luminal subtype with low levels of ZEB1/2 expression and high levels of E-cadherin expression ${ }^{8,15}$. Among the various HNSCC cell lines, we found that TSU and HOC313 cells, similar to MDA-MB-231 cells, express high levels of vimentin and ZEB1/2, and low levels of Ecadherin, while other HNSCC cells, similar to MCF7 cells, expressed high levels of E-cadherin and low levels of vimentin and ZEB1/2 (Fig. 1A, B and data not shown). Surprisingly, we found that $\mathrm{N}$-cadherin is expressed nearly ubiquitously in HNSCC cells used in this study.

Using reverse transcription-quantitative PCR (RTqPCR) analysis, we detected much higher levels of $Z E B 1 / 2$ mRNA in TSU and HOC313 cells than that in other epithelial-like HNSCC cells (Figs. 1C and S1A). Since we already reported that both ETS1 and ETS2 dramatically promote $Z E B 1$ promoter activation in breast cancer cells $^{10}$, we determined mRNA levels of ETS family genes by RT-qPCR analysis in HNSCC cells. ETS1 and ETS2 mRNAs were detected in all HNSCC cells without positive correlation with ZEB1/2 expression (Figs. 1C, D, and S1A). However, EHF mRNA expression profiles showed the strongest inverse correlation with ZEB1/2 in HNSCC cells (Fig. 1E). ELF3 mRNA levels also exhibited an inverse correlation with $Z E B 1 / 2$, though $E L F 5$ did not (Fig. 1E). These findings suggest that the EMT phenotypes of HNSCC cells are mediated by ZEB1/2, and that EHF regulates $Z E B 1$ expression without affecting mRNA levels of ETS in the mesenchymal-like HNSCC cells. In the Cancer Genome Atlas (TCGA) dataset of four oral squamous cell carcinoma tissues, $E H F$ mRNA levels were not correlated negatively with those of ETS1 (Fig. S1B), while a weak negative correlation was detected only in the GSE37991 dataset (Fig. S1B). Notably, EHF mRNA levels in all four datasets were significantly lower in cancer tissues compared to normal tissues, which was correlated with overall survival (Fig. S1C, D).

\section{Two EHF transcript variants with different $5^{\prime}$ UTRs}

Although $E H F$ is known to be similar in structure to $E L F 3$, it produces two transcript variants: a long form variant that includes the first exon, EHF-LF, and a short form variant that does not, EHF-SF (Fig. 2A). First, we examined which variants were expressed in HNSCC cells by conventional RT-PCR analysis. EHF-SF mRNA was detected in almost all HNSCC cells, whereas EHF-LF mRNAs were detected in several cell types, including SAS, 


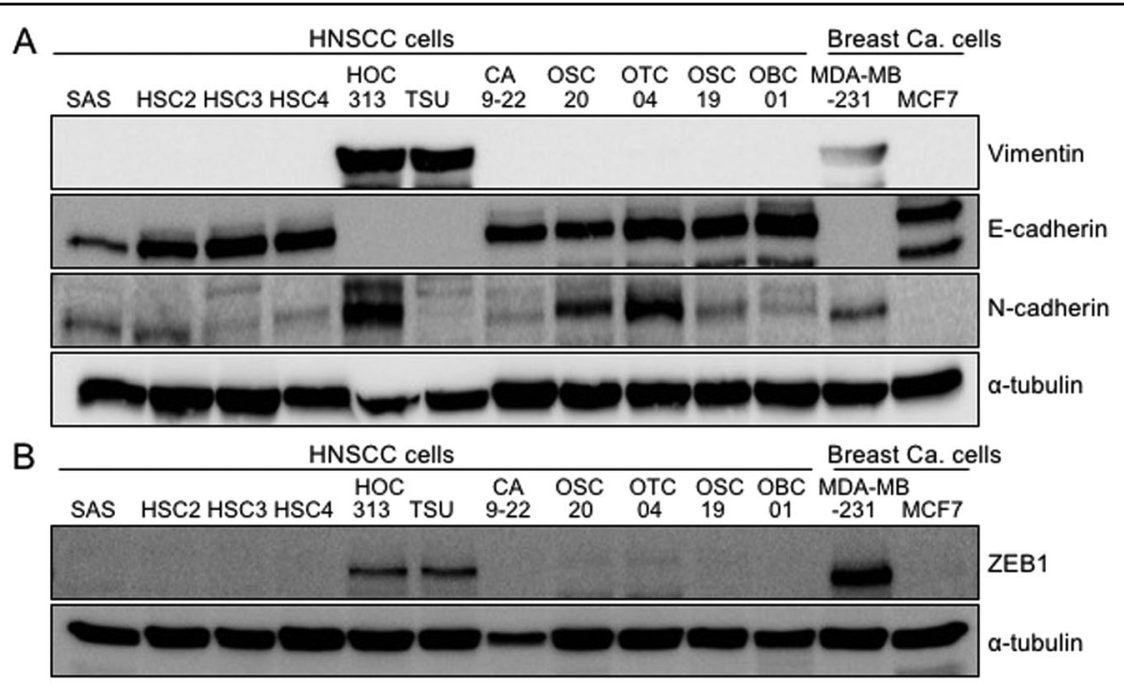

C
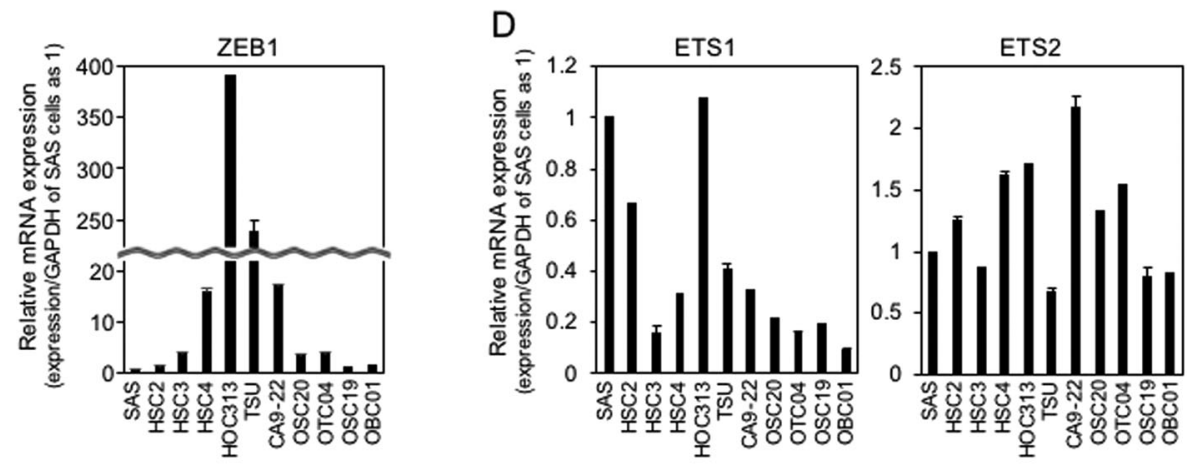

E

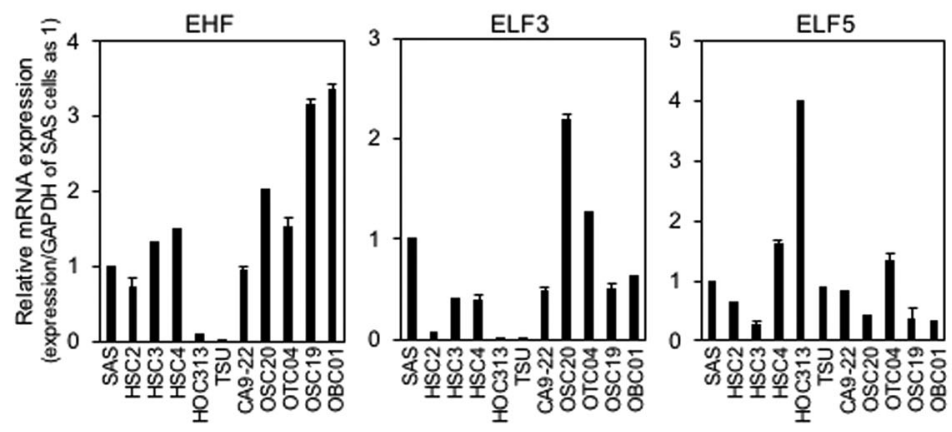

Fig. 1 EMT phenotypes in HNSCC cells. A, B, C, D, and E Representative markers of EMT in HNSCC cells were determined by IB and RT-qPCR. Breast cancer MDA-MB-231 and MCF7 cells were used as controls for ZEB1 expression levels. a-tubulin was used as a loading control (A and B). The ratio of each mRNA to GAPDH in SAS cells was indicated as "1" (C, D, and $\mathbf{E})$.

CA9-22, OSC20, OSC19, and OBC01 cells. Transcripts of both EHF variants were detected at low levels in TSU and HOC313 cells, respectively (Fig. 2B). Similar to the results of our RT-qPCR analysis (see Fig. 1E), total EHF levels were lower in mesenchymal-like HNSCC, TCU, and HOC313 cells (Fig. 2B). To determine whether the function of both variants is similar, we generated both EHF-LF and $E H F-S F$ cDNAs using mRNAs isolated from human cancer cells. After transfecting COS7 cells with the plasmids that encode either flag-tagged EHF-LF or EHFSF, we detected EHF-SF as a single band by SDS-PAGE and subsequent IB analysis using anti-flag and -EHF antibodies (Fig. 2C). By contrast, EHF-LF was detected with a few extra bands by both antibodies.

To exclude the possibility that flag-tagged EHF-LF generated a product that was translated starting from methionine 23 (Fig. 2A), Met 23 in EHF-LF was substituted with valine to generate EHF-LF (M23V). Similar 
A

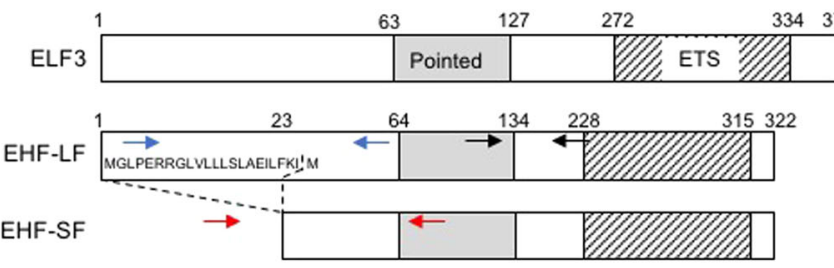

B
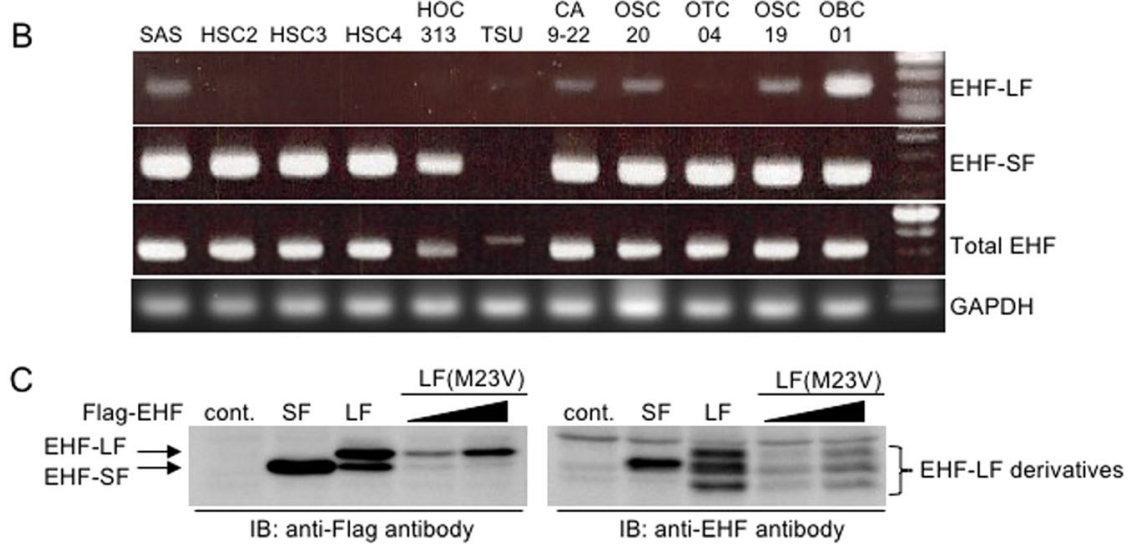

D
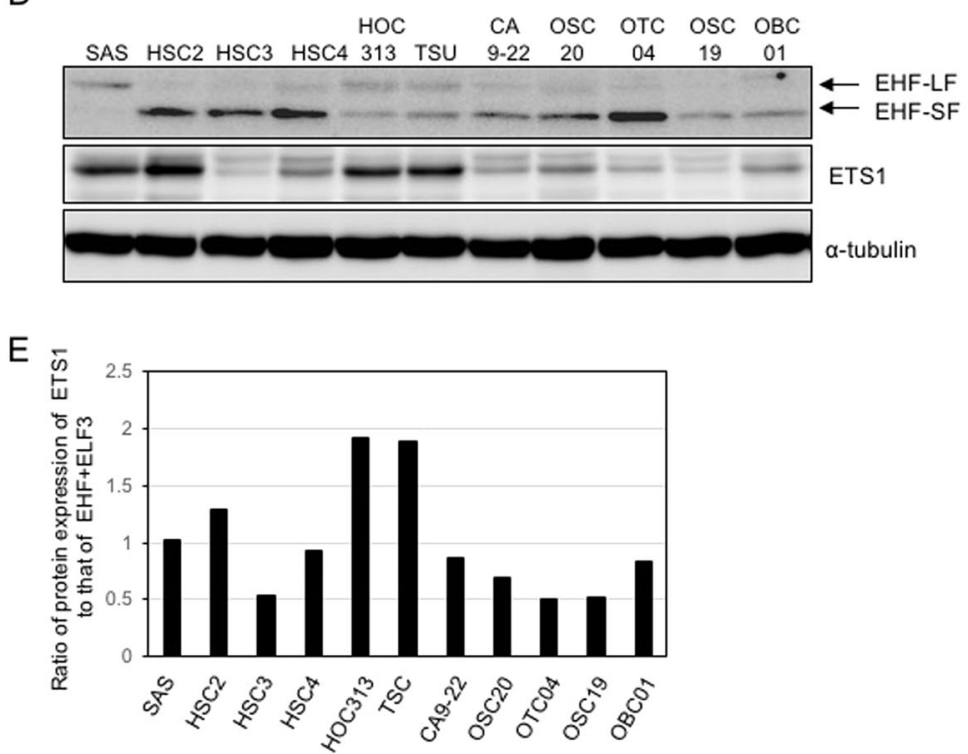

Fig. 2 Two variants of EHF. A Schematic illustration of ELF3 and EHF gene products is shown. The long form variant of EHF includes 22 amino acid residues from exon 1 (EHF-LF), whereas the short form variant of EHF excludes exon 1 (EHF-SF). The pointed and ETS domains are shown as grey and hatched areas, respectively. The primers used to amplify EHF-LF, EHF-SF, and total EHF are shown in blue, red, and black arrows, respectively. B mRNA levels of two EHF transcript variants were analyzed by conventional RT-PCR. C and D IB with the indicated antibodies were performed in COS7 transfected with control vector (cont.) or the indicated plasmids (C), and in HNSCC cells (D). E ETS1 (D), EHF-SF (D), and ELF3 (S2B) protein levels were densitometrically quantified and compared with those of a-tubulin. The ratio of protein expression of ETS1/a-tubulin to that of EHF-SF/a-tubulin + ELF3/a-tubulin in SAS cells was indicated as "1". a-tubulin was used as a loading control (D). EHF-LF(M23V), a mutant in which Met 23 in EHF-LF was substituted with $\mathrm{Val}(\mathbf{C})$.

to EHF-LF, EHF-LF(M23V) was detected as a few bands by IB analysis using anti-EHF antibody (Fig. 2C), suggesting that methionine residue at 23 was not available for translational start site to generate EHF-SF from the EHFLF mRNA.
Because the anti-EHF antibody recognized both EHF protein variants, we could determine expression of endogenous EHF in various HNSCC cells. SAS, HOC313, and TSU cells exhibited slowly migrated band, which was not consistent with EHF-LF mRNA expression in HNSCC 


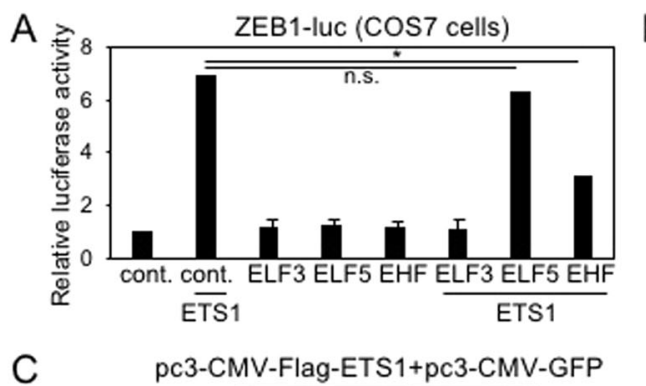

Flag-EHF - cont. LF SF -

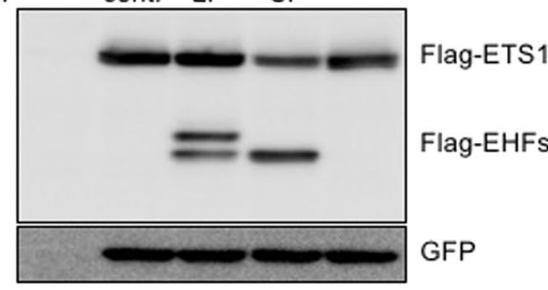

D

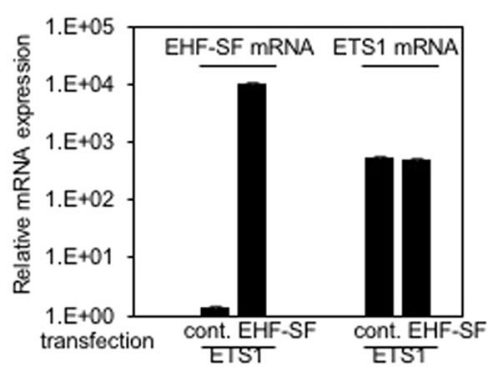

F

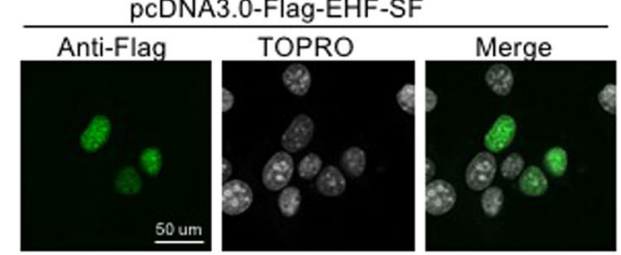

G

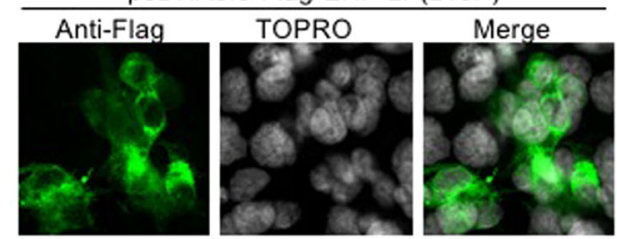

$\mathrm{H}$

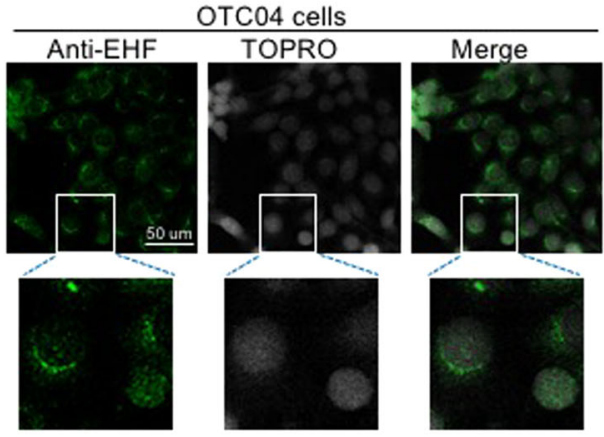

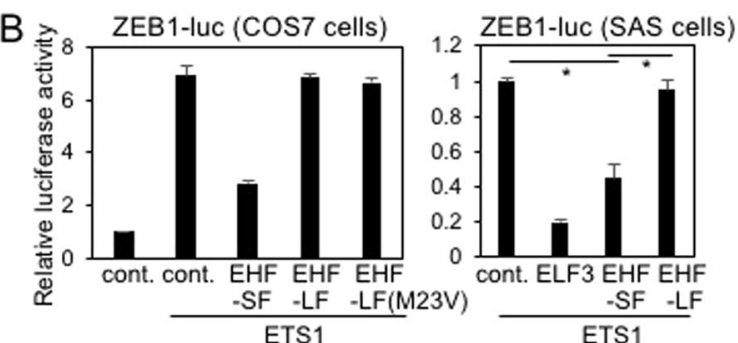

E
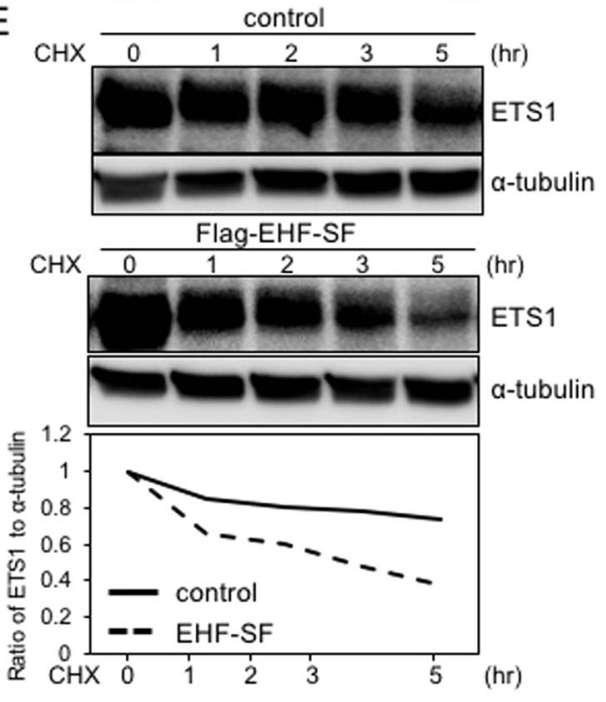

pcDNA3.0-Flag-EHF-LF

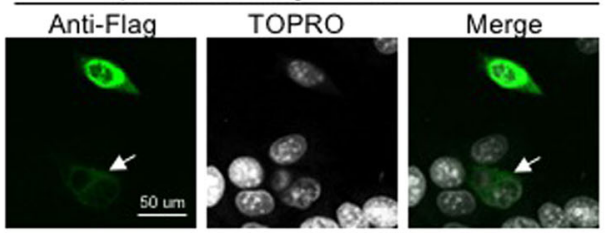

pcDNA3.0-Flag-EHF-LF(L11,15P)

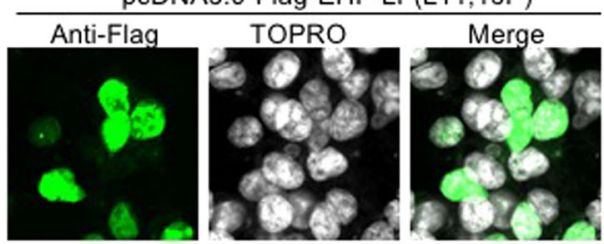

SAS cells
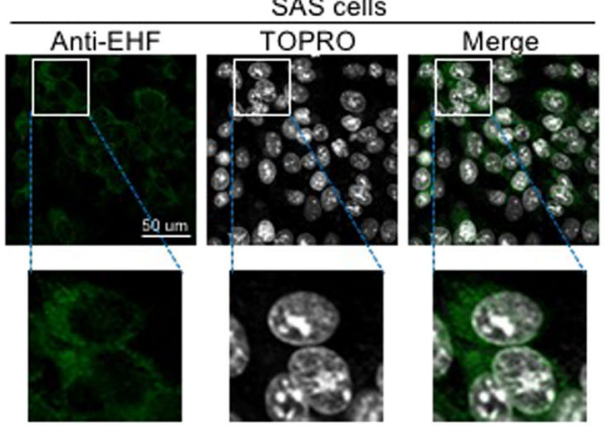

Fig. 3 (See legend on next page.) 
(see figure on previous page)

Fig. 3 Inhibitory effects of EHF variants on ZEB1 promoter activity. A and B ZEB1 promoter activities were determined by luciferase assays after transfection with control vector (cont.) or the indicated plasmids. Luciferase activity of cells transfected with the control vector was indicated as "1." Each value represents the mean \pm s.d. of triplicate determinations from a representative experiment. Similar results were obtained in at least three independent experiments. $p$ values were determined by Student's $t$-test. ${ }^{*} p<0.01$; n.s., not significant. (C and $\left.\mathbf{D}\right) \operatorname{COS7}$ cells were transfected with the indicated plasmids, followed by IB (C) and RT-qPCR (D) to determine the levels of transfected genes. Ectopic EHF-SF mRNA levels of cells transfected with ETS1 alone was indicated as "1" (D). E COS7 cells pre-transfected with flag-tagged ETS1 were further transfected with either control vector or flag-EHF-SF plasmid, and treated with cycloheximide (CHX) for the indicated time, followed by IB. The ratio of ETS1 to a-tubulin was determined by densitometric analysis and shown at the bottom. (F and $\mathbf{G}$ ) COS7 cells were transfected with the indicated plasmids followed by

immunofluorescence analysis using an anti-flag antibody. Arrow indicates cells with low expression of flag-EHF-LF. H Subcellular localization of endogenous EHF was determined by anti-EHF antibody in HNSCC OTCO4 and SAS cells. High magnification is shown in bottom panels.

cells (Fig. 2B, D). Thus, we confirmed it by specific siRNAs against EHF (Fig. S2A), suggesting that the upper band in these cells was derived from EHF-LF. OTC04, HSC2, HSC3, and HSC4 cells expressed EHF-SF at high levels (Fig. 2D). Both mesenchymal-like HOC313 and TSU cells, which express high levels of ETS1 protein, expressed low levels of EHF and ELF3, (Figs. 1E, 2D and S2B), suggesting that the EMT-like phenotypes are highly dependent on the ratio of ETS1 protein levels to EHF and ELF3 protein levels (Fig. 2E).

\section{Only the EHF-SF variant suppresses ZEB1 promoter activity}

To examine whether EHF affects ZEB1 promoter activity, we transfected cells with plasmids carrying $E H F$ $S F, E H F-L F$, as well as ELF3 in combination with ETS1 plasmid, and determined $Z E B 1$ promoter reporter activity thereafter. As previously reported ${ }^{10}$, ETS1 dramatically activated the promoter's activity, which was almost completely inhibited by ELF3 (Fig. 3A). EHF also partially inhibited the promoter activity induced by ETS1, whereas ELF5 failed to do so. Surprisingly, EHF-LF did not affect ZEB1 promoter activity induced by ETS1, which was also observed in HNSCC SAS cells (Fig. 3B). EHF-LF (M23V) behaved similarly to EHF-LF, because Met 23 was not used as an initiation codon for translation to produce EHF-SF (Fig. 3B and see Fig. 2C). So far, it remains unclear how ELF3 inhibits ZEB1 promoter activation by ETS1 in breast cancer cells ${ }^{10}$. To investigate the inhibitory effects of EHF-SF, we transfected EHFs together with both GFP and ETS1, both of which were subcloned into the same expression vectors under the control of the CMV promoter (pcDNA3.0). Overexpression of EHF-SF downregulated ETS1 protein levels in cells; however, overexpressed EHF-SF did not affect GFP protein levels. We also determined that EHF-SF downregulated ETS1 protein levels without affecting ETS1 mRNA levels, whereas EHF-LF failed to affect either (Fig. 3C, D). In addition, we determined through experiments using cycloheximide that EHF-SF shortens the half-life of ETS1 proteins (Fig. 3E). We also found that siRNAs against $E H F$ upregulated endogenous ETS1 proteins (Fig. S2C), which suggests that EHF-SF promotes the degradation of ETS1 proteins. Moreover, overexpressed ETS1 and ETS2 were downregulated in a manner dependent on the amount of EHF expression plasmids used for transfection (Fig. S2D).

Leucine-rich peptides are known to act as nuclear export signal/sequence (NES) ${ }^{16}$. We found that the first exon in EHF-LF is composed of 22 amino acid residues, including seven leucine and two isoleucine residues (see Fig. 2A). Upon transfection, EHF-SF and EHF-LF were clearly localized to the nucleus and cytoplasm, or to the perinuclear region, respectively, as determined by immunofluorescence using an anti-flag antibody (Fig. 3F). When leucine residues in the first exon of EHF-LF were substituted with proline residues, a double point mutant (L11,15P), but not a single point mutant (L15P), exhibited nuclear localization very similar to that of EHF-SF (Fig. 3F, G). This similarity suggests that leucine-rich residues in the first exon of EHF-LF regulate EHF subcellular localization. Also, the subcellular localization of EHF-LF in SAS cells differed slightly from that of EHF-SF in OTC04 cells (Fig. 3H). When the NES of EHF-LF was fused to EHF-SF (EHF-nesSF), the fusion protein was localized to the perinuclear cytoplasm and failed to degrade ETS1 proteins (Fig. S2E, F). Leptomycin B inhibits CRM1 (chromosomal region maintenance)/exportin 1 , a protein required for nuclear export of proteins containing an $\mathrm{NES}^{17}$. Following treatment with leptomycin B, nuclear localized EHF-LF partially promoted the degradation of ETS1 proteins (Fig. S2E, G), suggesting that EHF nuclear localization involves degradation of ETS proteins.

\section{Biological significance of EHF in HNSCC cells}

We next addressed the biological effects of EHF in HNSCC cells. At first, after TSU cells were infected with EHF-LF lentiviruses, we had difficulty detecting the EHFLF protein despite the presence of the corresponding mRNA. The protein only became detectable following treatment with MG132, a proteasome inhibitor (Fig. S3A). In addition, endogenous EHF-LF proteins were upregulated in epithelial-like HNSCC cells following MG132 treatment (Fig. S3B). By contrast, when lentiviruses encoding EHF-SF were used for transduction, EHF-SF proteins were detected at moderately high levels even in 


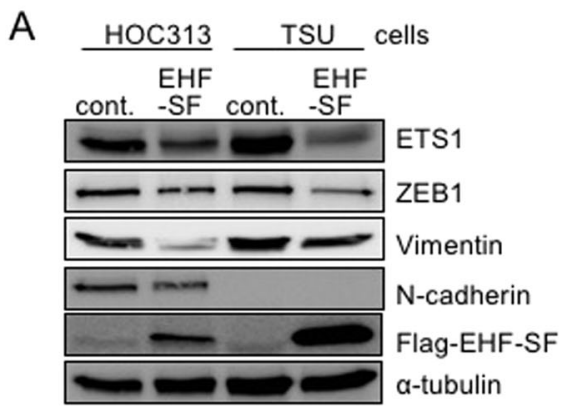

C

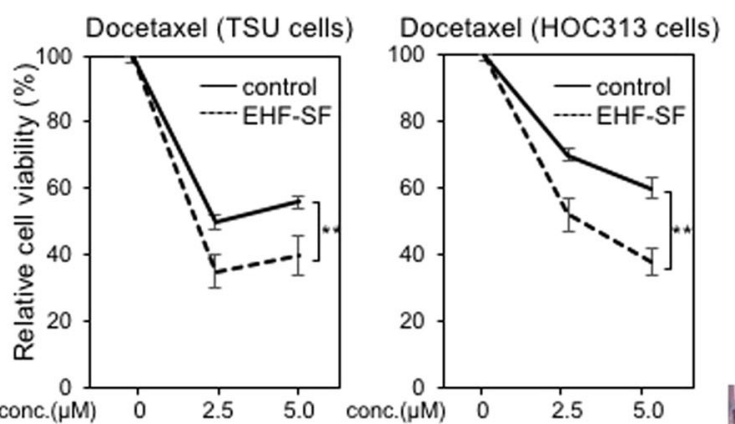

D

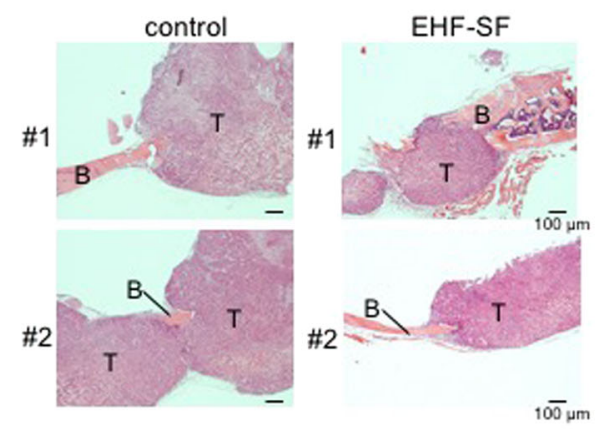

E

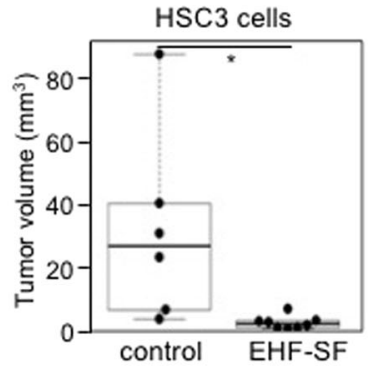

I

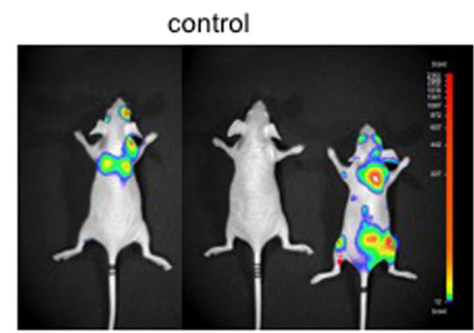

B
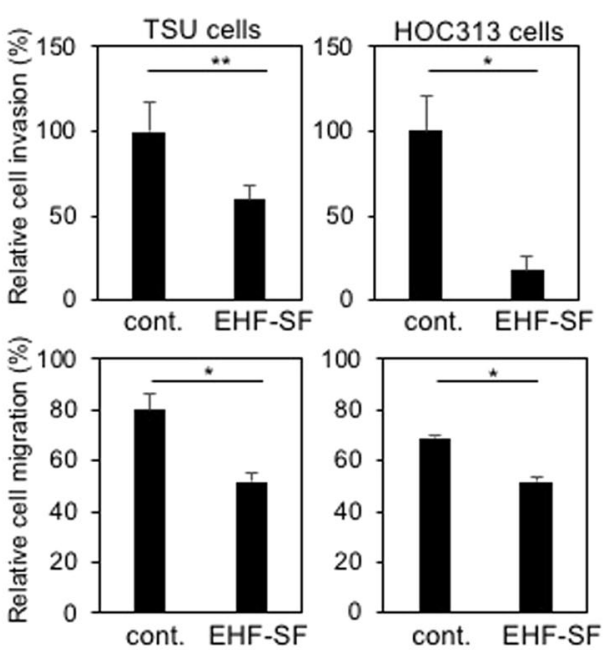

F

G

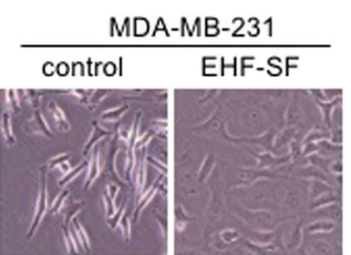

MDA-MB-231 cells control EHF-SF

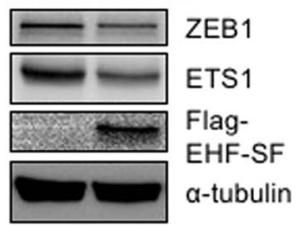

$\mathrm{H}$

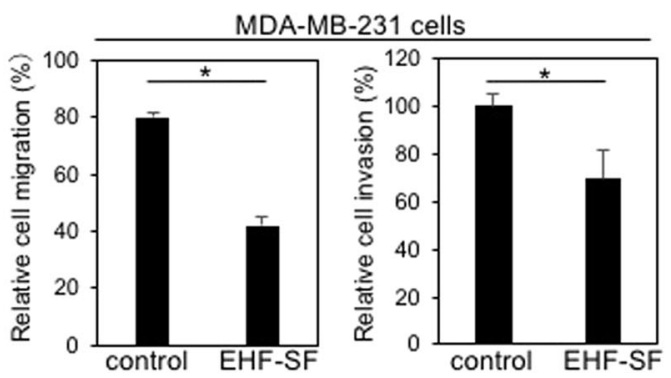

J
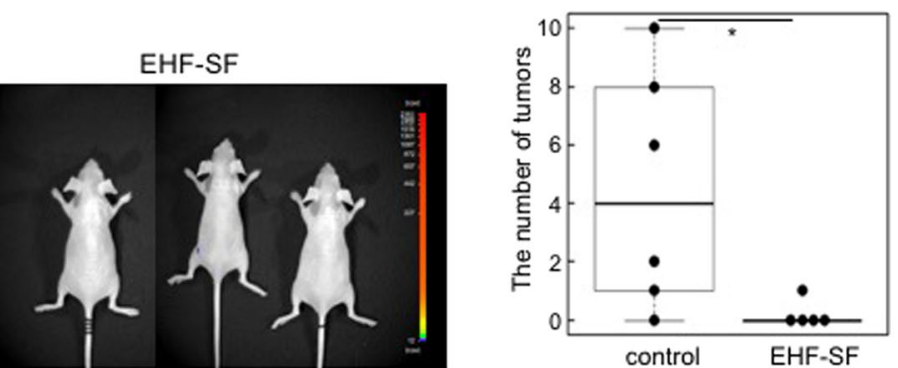

Fig. 4 (See legend on next page.) 
(see figure on previous page)

Fig. 4 Inhibition of oncogenic effects of ETS1 by EHF-SF. A, B, and C HOC313 and TSU cells infected with lentiviruses carrying control (cont.) or flag-tagged EHF-SF were subjected to IB analysis using the indicated antibodies (A). Motility assays using a Boyden chamber assay with inserts coated with type I collagen gel (upper panels) and a wound healing assay (lower panels) (B). Chemoresistance assay in response to the indicated concentration (conc.) of docetaxel $(\mathbf{C})$. Each value represents the mean \pm s.d. of triplicate determinations from a representative experiment. Similar results were obtained in at least three independent experiments. D and E HSC3 cells infected with either control $(n=6)$ or flag-tagged EHF-SF ( $n=$ 8) were injected into the periosteal region of the parietal bone in mice. Five weeks later, mice were sacrificed, and tumor volume was measured (E). After decalcification, specimens were prepared. Typical histology in hematoxylin and eosin staining are shown. B bone, T tumor. (F, G, H, I, and $\mathbf{J})$ MDA-MB-231-Luc cells infected with either control or EHF-SF were visualized by phase-contrast microscopy (F), and subjected to IB analysis (G) and motility assays $(\mathbf{H})$. The cells were injected into the left ventricle of the heart, followed by imaging analysis $(\mathbf{I})$ and quantification $(\mathbf{J})$ at 35 days (5 weeks). $p$ values were determined by Student's t-test. ${ }^{*} p<0.01,{ }^{* *} p<0.03$. a-tubulin was used as a loading control (A and $\left.\mathbf{G}\right)$.

the absence of MG132. MG132 treatment caused EHF-SF protein levels to increase slightly likely due to upregulation of its mRNA levels (Fig. S3C). Based on the finding that endogenous EHF-SF was easily detected by IB analysis in HNSCC cells (see Fig. 2D), endogenous EHF-SF protein is more stable in the nucleus than EHF-LF.

Following infection with EHF-SF, EHF-SF proteins were detectable in mesenchymal-like HOC313 and TSU cells in the absence of MG132 (Fig. 4A). In these lentivirus-infected cells, endogenous expression of both ETS1 and ZEB1 was repressed. In addition, vimentin was also downregulated by EHF-SF in both cell types, while $\mathrm{N}$-cadherin was also repressed in HOC313 cells and barely detectable in TSU cells (Fig. 4A). Motility was suppressed by EHF-SF in both cell types according to migration and Boyden chamber assay, which was enhanced by sublethal concentration of docetaxel, an anti-cancer drug frequently used as a clinical therapeutic agent for patients with head and neck cancer (Figs. 4B, S3D, E) ${ }^{18}$. Sensitivity to docetaxel was significantly increased (Figs. $4 \mathrm{C}$ and S3F). Thus, these findings suggest that EHF inhibits EMT phenotypes, including motility and anti-cancer drug resistance, to some extent in HNSCC cells.

In vivo tumor growth was determined by a xenograft model involving transplantation of HSC3 cells into the calvarial region of mice ${ }^{19}$. Five weeks after transplantation, in vivo tumor growth was reduced by EHF-SF (Figs. $4 \mathrm{D}, \mathrm{E}$ ), while in vitro cell growth was not altered by EHFSF overexpression in HSC3 cells (Fig. S3G). Since changes in the infiltration of the cells into calvaria (bone destruction) were not clearly evaluated by this experimental model, we went on to use breast cancer MDAMB-231 cells to determine in vivo metastasis after intracardiac injection. We also chose this breast cancer cell line, because all the HNSCCs used in this study do not metastasize sufficiently to distant organs in mice. When EHF-SF was overexpressed in MDA-MB-231 cells, the cell morphology was altered from spindle-like to cobblestonelike shapes, a change that was accompanied by repression of ZEB1 and ETS1 as well as in vitro cell migration and anti-cancer drug resistance (Figs. 4F, G, H, and S3H). In vivo metastasis was also repressed by EHF-SF (Fig. 4I, J), suggesting that EHF-SF ameliorates the exacerbation of cancer in vivo.

\section{Tumor promotion in EHF-silenced cells}

To perform loss of function experiments, we isolated several monoclones of HNSCC OBC01 cells after endogenous $E H F$ was knocked out by CRISPR/Cas9 techniques using commercially available gRNA against the $E H F$ gene. Although endogenous EHF-SF in these monoclones failed to be completely disrupted as determined by IB analysis, two monoclones exhibited moderate downregulation of EHF-SF and slight upregulation of ZEB1, leading to enhancement of motility and docetaxel resistance (Fig. 5A, B, C). In addition to the knockout cells, we generated $\mathrm{OBC} 01$ and $\mathrm{HSC} 2$ cells in which endogenous EHF expression was knocked down by specific siRNAs against $E H F$. The cells transfected with the siRNAs exhibited induction of ZEB1 mRNA and ZEB1 protein expression. ETS1 protein levels were also increased without drastically affecting ETS1 mRNA levels (Figs. 5D, E, and S4A). In addition, susceptibility to docetaxel was also reduced by EHF-specific siRNAs (Fig. 5F).

Immunohistochemical analysis was also performed using an anti-EHF antibody on specimens from human patients with tongue cancer after we characterized the specificity of the antibody using cells transfected with either siRNAs against EHF or plasmid encoding EHF-SF (data not shown). In the neighboring healthy tissues of patients with tongue cancer, EHF was predominantly stained in the nuclei in the cells of stratum spinosum, and hardly observed in basal layer cells (Fig. 5G, left in top panels). Some of the lymphoid cells also stained positive. In carcinoma tissues, EHF was observed in the nuclei of almost all cells in the tumor nest (Fig. 5G, right in top panels). Along with the invasion front, some cancer cells were either unstained or stained, coexisting with other cells that stained positive (Fig. 5G, bottom panels). Notably, statistical analysis showed that EHF was significantly reduced in cells at invasion front in high grade HNSCC (Fig. 5H). Therefore, EHF-SF acts as an anti-tumor promoting factor or anti-EMT factor in cancer. 

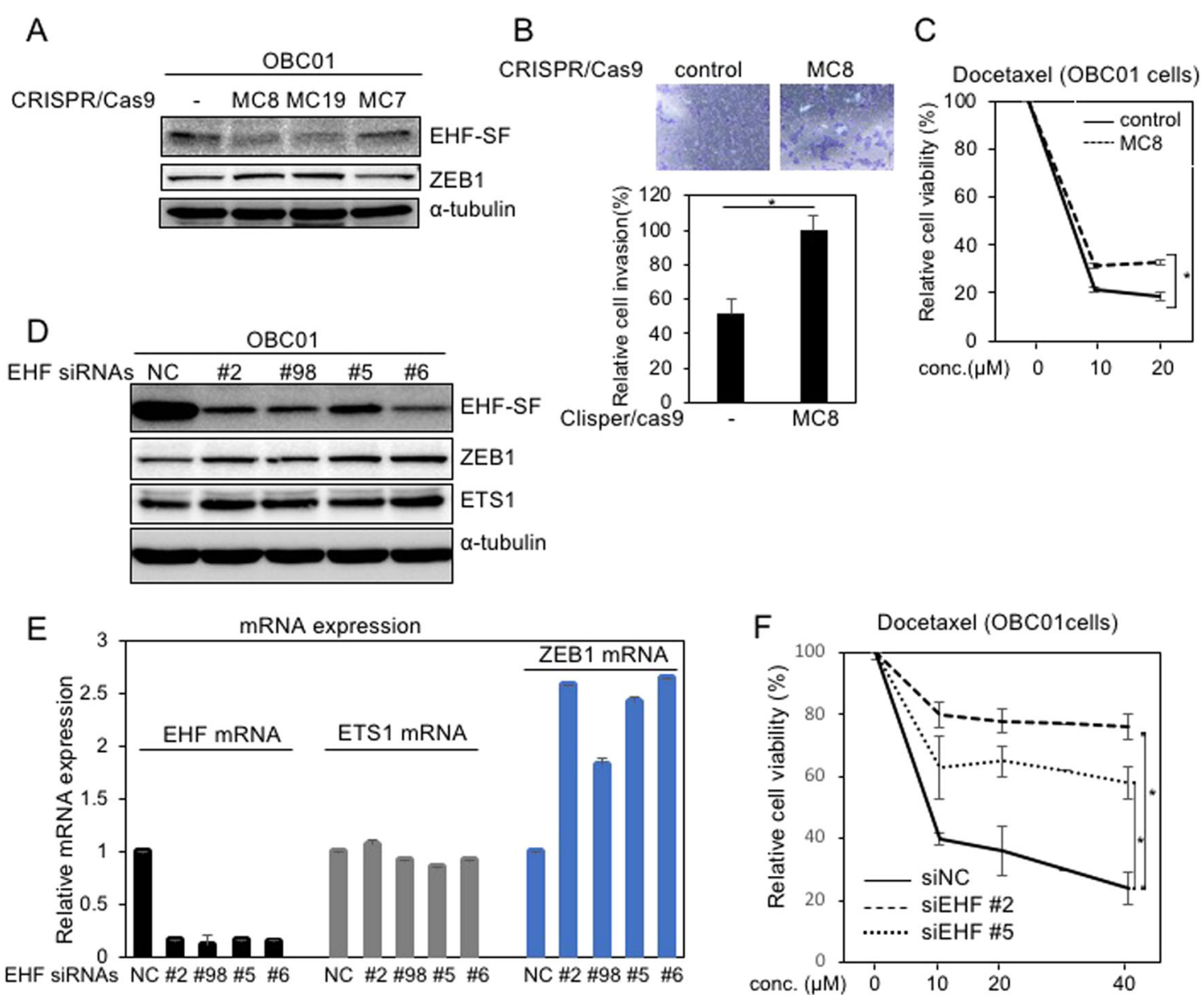

G

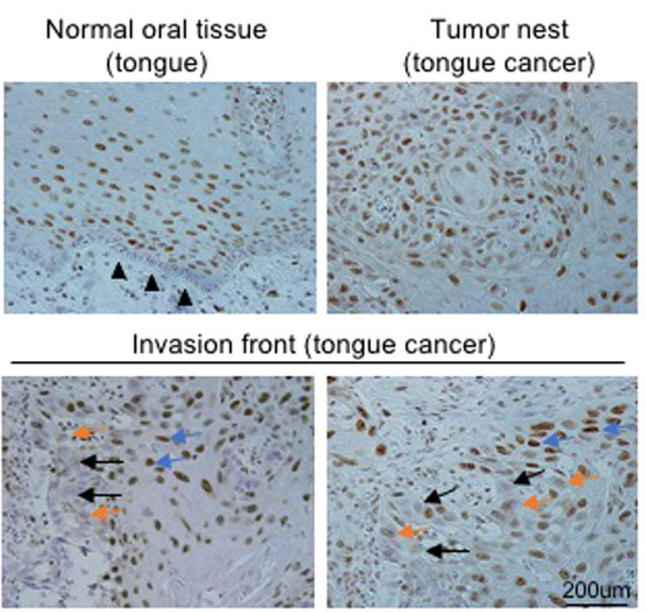

$\mathrm{H} \quad$ EHF expression at invasion front

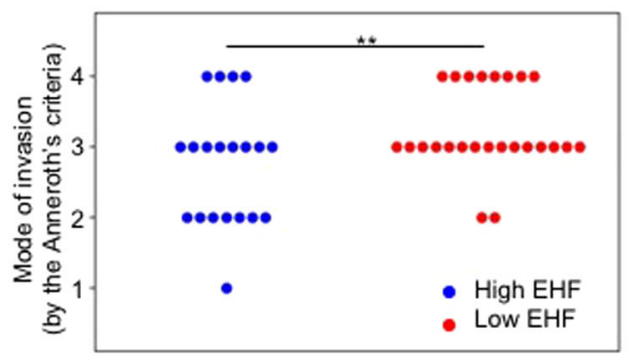

Fig. 5 (See legend on next page.)

Identification of a novel point mutation in the EHF gene in cancer cells

When we generated $E H F$ cDNAs using mRNAs isolated from breast cancer cells by RT-PCR in this study, we found that a leucine residue at 285 in EHF was mutated to proline (L285P) in several plasmid clones purified from bacteria. Proline 285 was restored to leucine by mutagenesis for use in the present experiments. Interestingly, we found that the region around position of 285 in ELF3, but not EHF, is a region where amino acid residues are 
(see figure on previous page)

Fig. 5 Effect of gene silencing of EHF in HNSCC cells. A, B, and C After knocking out the indicated genes by CRISPR/Cas9 techniques, monoclones were established and examined by IB analysis $(\mathbf{A})$, invasion assays $(\mathbf{B})$, and chemoresistance assays in response to the indicated concentration (conc.) of docetaxel in OBC01 cells (C). D, E, and $\mathbf{F}$ OBC01 cells transfected with control siRNA (NC) or EHF siRNAs were subjected to IB analysis (D), RT-qPCR analysis $(\mathbf{E})$, and chemoresistance assays in response to the indicated concentration (conc.) of docetaxel (F). The ratio of mRNA expression to GAPDH in cells transfected with control siRNA (NC) was indicated as "1" (E). Each value represents the mean \pm s.d. of triplicate determinations from a representative experiment. Similar results were obtained in at least three independent experiments. $p$ values were determined by Student's $t$-test. ${ }^{*} p$ $<0.01$ ( $\mathbf{C}$ and $\mathbf{F}$ ). a-tubulin was used as a loading control ( $\mathbf{A}$ and $\mathbf{D})$. $\mathbf{G}$ and $\mathbf{H}$ Representative images of immunohistochemical staining with anti-EHF antibody are shown in normal tongue tissue (top left panel), tumor nest (top right panel), and the invasion front (bottom two panels). Arrowheads indicate basal layer cells in normal tissue. Black arrows, orange arrows, and blue arrows represent EHF-negative, EHF-weak, and EHF-positive cancer cells at the invasion front, respectively $(\mathbf{G})$. After representative images at the invasion front were randomly selected, we evaluated EHF-positive or negative/weak cancer cells at the invasion front, which were further assessed according to the Anneroth's criteria by pathologists (H). Student's $t$-test was used to compare differences between groups. ${ }^{* *} p<0.02$.

frequently mutated in ampullary carcinoma (Fig. 6A) $)^{12}$. Since the L329P mutation in ELF3 is deposited in the TCGA dataset for bladder cancer (Fig. 6A), the corresponding L285P mutation in EHF was further investigated. Most surprisingly, the L285P mutation in EHF-SF abolished the effects of wild-type EHF-SF without affecting subcellular localization (Fig. 6B, C, D). We also constructed R287G and A288P mutants of EHF, which correspond to R331G and A332P, respectively, in the ELF3 gene mutation in ampullary carcinoma (Fig. 6A) ${ }^{12}$. In addition, we constructed the R287* mutant of EHF, which is a nonsense mutation at position 287 deposited in the glioma TCGA dataset. The A288P and R287* mutations in EHF-SF failed to inhibit ETS1-induced activation of the ZEB1 promoter and degrade ETS1, while the R287G mutant functioned similar to wild type EHF-SF (Figs. 6D, E, and S4B). When overexpressed from lentiviral vectors in TSU and HOC313 cells, which express endogenous EHF-SF only at low levels (see Fig. 2D), the EHF-SF-L285P mutation no longer downregulated ETS1 and $Z E B 1$, inhibited motility, and sensitized cells to anticancer drug (Fig. 6F, G, H). These finding strongly suggest that the L285 mutation results in loss of anti-EMT capacity of EHF-SF to inhibit EMT phenotypes.

\section{Tumor promoting effects of EHF-SF-L285P in vivo}

ELF3 mutations are estimated to be a probability of heterozygous loss of function ${ }^{12}$. To determine if this is also a property of $E H F$, we first performed a titration analysis using expression plasmids encoding EHF-SF and EHF-SF-L285P in combination with the ETS1 plasmid. Increased amounts of EHF-SF-L285P overcame the inhibitory effect of EHF-SF (Fig. 7A), which suggested that a dominant negative effect may be at work. Thus, we transduced epithelial-like HNSCC HSC4 and OTC04 cells, both of which express endogenous EHF-SF (see Fig. 2D), with EHF-SF-L285P lentiviruses. EHF-SF-L285P overexpression caused an increase in ETS1 and ZEB1 expression and promoted both migration and chemoresistance in both cell types (Fig. 7B, C, D). Cell proliferation in vitro was not dramatically affected by overexpression of EHFSF-L285P (Fig. 7E). We next investigated the role of the L285P mutation in bone invasion into the calvarial region in a xenograft transplantation model. Unlike HSC3 cells (see Fig. 4), HSC4 cells are known to be less effective in bone invasion in this model than HSC3 cells (unpublished data). Five weeks after transplantation, HSC4 cells infected with EHF-SF-L285P exhibited enhanced tumor growth, compared to control cells. Histochemical analysis showed that control HSC4 cells adhered to the calvarial bone through periosteum-like mesenchymal tissues, while EHFSF-L285P overexpression caused direct invasion into the bone and promoted tumor growth (Fig. 7F, G). These effects of EHF-SF-L285P were also determined using experimental pulmonary models with osteosarcoma 143B cells. After the cells were injected into the tail vein, the number of nodules in lung tissues increased with EHF-SFL285P (Fig. 7H). These findings suggest that EHF-SFL285P promotes tumor growth and invasion in vivo.

We further explored EHF mutations in human genomic DNA. Human genomic DNA was randomly extracted from 15 patients with tongue cancer by microdissection and analyzed by next generation sequencing of the ETS domain-encoding sequence in $E H F$. Unfortunately, we did not detect the L285P mutation, but we did detect mutations with missense, nonsense, and silent mutations in this region (Fig. S5). Notably, mutations in ELF3 were not detected in these samples. Thus, EHF-SF, rather than ELF3, is associated with cancerization and progression of HNSCC.

\section{Discussion}

In this study, we elucidated the functional differences between two EHF variants in cancer cells. Only EHF-SF, which is more widely expressed in HNSCC cells, can promote the degradation of ETS proteins, and, in turn, downregulate ZEB1/2, the most representative among EMT transcription factors (EMT-TFs). Therefore, we propose that EHF functions as an anti-EMT factor. Most importantly, the L285P mutation in EHF-SF abolishes its 
A

L329P(bladder Ca in TCGA)

I. A332P(ampullary Ca)

ELF3 AVAQLWGQKKKNSNMTYEKLSRAMRYYYKREILERVDGRRLVYKF

L285P(breast Ca cell line)

1 R287*(brain glioma in TCGA)

AVAQLWGKKKNNSSMTYEKLSRAMRYYYKREILERVDGRRLVYKF (H.S)

EHF AVAQLWGKKKNNSSMTYEKLSRAMRYYYKREILERVDGRRLVYKF (M.M)

AVAQLWGKKKNNNSMTYEKLSRAMRYYYKREILERVDGRRLVYKF (C.L.)

C

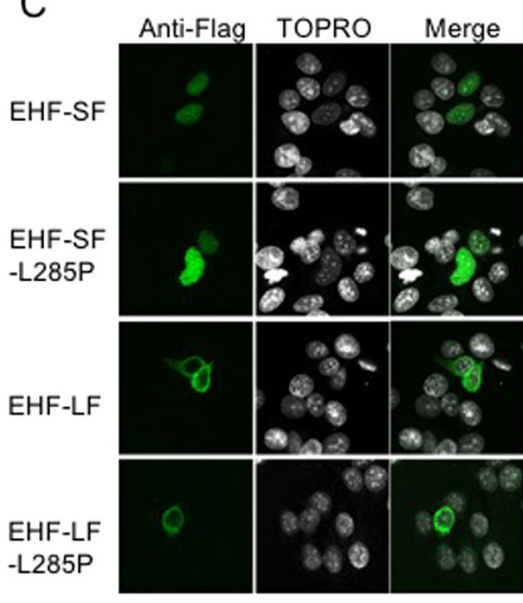

F

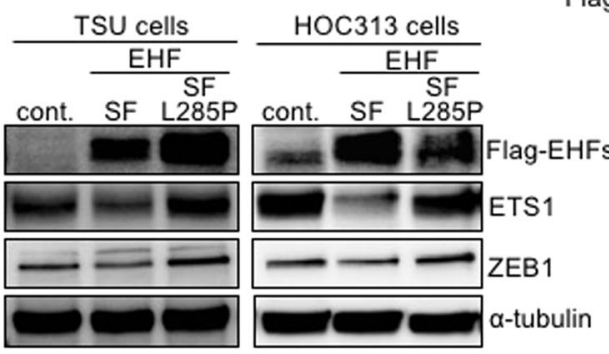

G
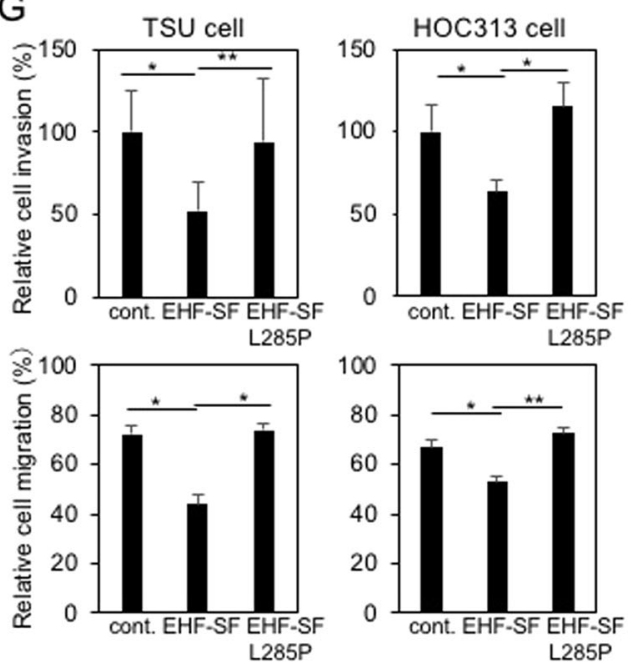

\section{B pc3-CMV-Flag-ETS1 + pc3-CMV-GFP \\ Flag-EHF cont. LF LF SF SF L285P L285P}

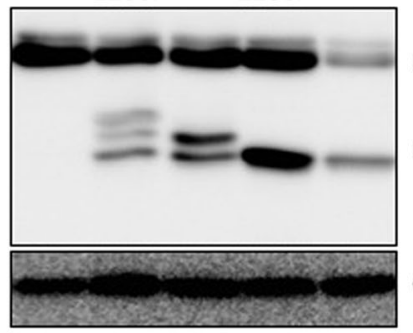

Flag-ETS1

Flag-EHFs

GFP

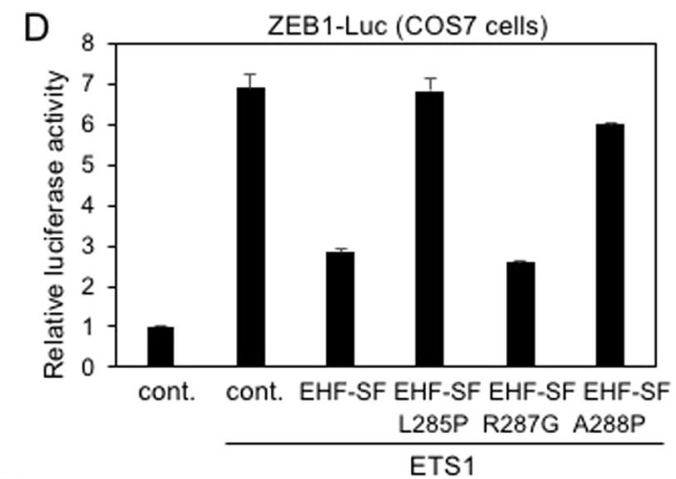

E

Flag-ETS1

Flag-EHF - $\quad$ SF SF LF LF SF SF

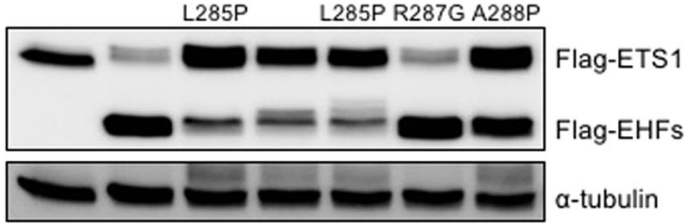

$\mathrm{H}$

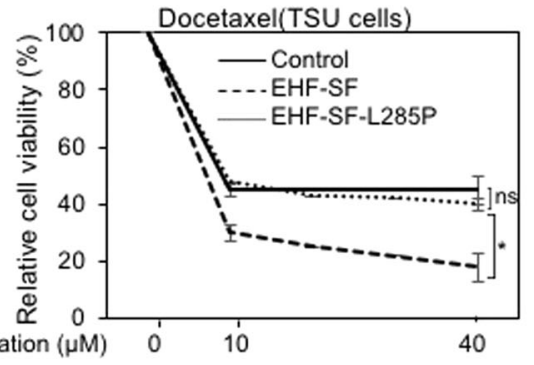

Concentration $(\mu \mathrm{M}) \quad 0 \quad 10$

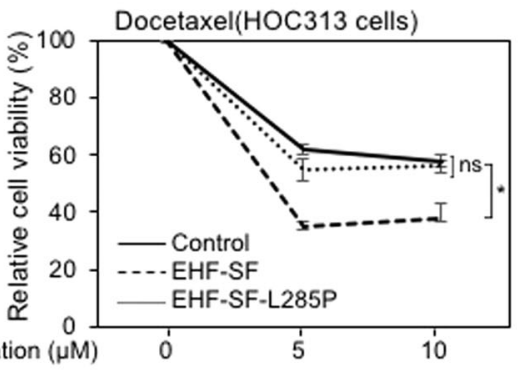

Fig. 6 (See legend on next page.) 
(see figure on previous page)

Fig. 6 Identification of a point mutation in the EHF gene. A Amino acid comparison of a part of the ETS domain is shown in ELF3 (top) and EHF (bottom). Arrowheads indicate mutation previously annotated in the TCGA dataset or previous reports. Identification of a novel L285P mutation in EHF in breast cancer cells indicated with a red arrow. H.S Homo sapiens, M.M Mus musculus, C.L Canis lupus. B, C, D, and E Expression plasmids for EHF and its derivative mutants were transfected into COS7 cells and examined by IB (B and $\mathbf{E})$, immunofluorescence $(\mathbf{C})$, and luciferase analysis $(\mathbf{D})$. $\mathbf{F}, \mathbf{G}$, and $\mathbf{H}$ TSU and HOC313 cells were infected with lentiviruses carrying control (cont.), flag-tagged EHF-SF, or flag-tagged EHF-SF-L285P, and subjected to IB analysis (F), motility assays using Boyden chamber assay with inserts coated with type I collagen gel (upper panels) and wound healing assays (lower panels) $(\mathbf{G})$, and chemoresistance assays $(\mathbf{H})$. Each value represents the mean \pm s.d. of triplicate determinations from a representative experiment. Similar results were obtained in at least three independent experiments. $p$ values were determined by Student's $t$-test. ns not significant; ${ }^{*} p<0.01,{ }^{* *} p<0.03$. a-tubulin was used as a loading control $(\mathbf{B}, \mathbf{E}$, and $\mathbf{F})$.

function and permits ETS1-mediated ZEB1/2 expression to promote EMT during cancer progression.

\section{Identification of the ESE domain in the anti-EMT function in cancer cells}

The C-terminus of EHF (a.k.a. ESE3) contains an ETS domain (Fig. S5), though its amino acid sequence is only about $40 \%$ similar to that of ETS1. Hereafter, we call it the "ESE" domain, because ESE domain of EHF bears $85 \%$ amino acid similarity to that of ELF3 (a.k.a. ESE1) and 80\% similarity to that of ELF5 (a.k.a. ESE2), respectively. A number of mutations in ELF3 were identified in ampullary carcinoma by next generation sequencing analysis, many of which occur in the ESE domain, referred to as a hotspot region. Thus, ELF3 is now known to be an anti-oncogene with potential haploinsufficiency ${ }^{12}$. Likewise, mutations in ELF3 in many cancer tissues have also been deposited in the TCGA dataset, whereas those in EHF have not. In this study, we identified an EHF mutation that lies in the ESE domain (L285P) in breast cancer cells, which corresponds to the L329P mutation in ELF3 in bladder carcinoma (Fig. 6A). We also found mutations in genomic DNA within the ESE domain-encoding region of EHF in patients with tongue cancer (Fig. S5), which have not been deposited in TCGA or other databases. In addition, mutations around the L285 position in EHF were also deposited in the glioma TCGA dataset (Fig. 6A).

Notably, the molecular mechanism how ELF3 mutants act as anti-oncogenes has not been elucidated so ar $^{12}$; however, we discovered that EHF-SF promotes degradation of the oncogene products ETS1 and ETS2, and subsequently reduces expression of ZEB1/2, acting as an anti-EMT factor. By contrast, the L285P mutation in EHF-SF impairs its intrinsic functions, suggesting that the ESE domain is indispensable for the anti-EMT effects of EHF-SF. Similar to EHF, ELF3 inhibited ZEB1 promoter activation by ETS1 (Fig. 3A), and also promoted the degradation of ETS proteins. The L329P mutation, which corresponds to L285P in EHF, abolished its original function (Fig. S6A).

\section{Molecular mechanism of ETS1 degradation by EHF-SF}

The precise molecular mechanism by which EHF-SF promotes ETS1 degradation remains unclear. ETS1 is known to be phosphorylated at specific serine and threonine residues by ERKs and CaMKII, some of which promotes ubiquitination by COP1 and sumoylation by UBC9 and $\mathrm{PIAS}^{20}$. Phosphorylation of a neighboring tyrosine residue by Src family kinases can disrupt binding of COP1, stabilizing ETS1 ${ }^{21}$. In our study, after immunoprecipitated ETS1 was blotted with anti-phosphotyrosine antibody (Py20), phosphorylated tyrosine was not clearly detected in either the presence or absence of EHF-SF or EHF-SF-L285P (data not shown). In addition, ETS1 degradation by EHF-SF was not blocked by several inhibitors against src (PP1), MEK1/2 (U0126), CaM kinase II (KN93), and PKC (Gö 6983) (Fig. S6F). A sumoylation inhibitor (2-D08) and lysosome inhibitor (chloroquine) also did not block ETS1 degradation (Fig. S5E and data not shown) ${ }^{22}$. In contrast, the proteasome inhibitors, MG132 and Lactacystin, inhibited ETS1 degradation induced by EHF-SF (Fig. S6B). In cells transfected with siRNAs against $E H F$, the stabilizing effects of MG132 on endogenous ETS1 protein were reduced (Fig. S6C), suggesting that endogenous EHF constitutively reduces ETS1 protein levels by proteasomaldependent degradation. MG132 is a potent proteasome inhibitor with trileucine peptides (N-Benzyloxycarbonyl-Lleucyl-L-leucyl-L-leucinal), suggesting that the leucine repeat sequence (LVLLLSL) in the first exon of EHF-LF would behave like MG132. Thus, EHF-LF would act as a bait against unidentified molecules that degrade ETS proteins, resulting in stabilization of ETS proteins.

\section{Genomic alteration of the EHF gene}

When we isolated EHF cDNAs from mRNAs of breast cancer cells, we accidentally found that a leucine residue at 285 in EHF was mutated to proline (L285P) in several clones. We therefore investigated genomic alteration in the ESE domain of the EHF and ELF3 genes by sequencing, after genomic DNA was isolated from specimens of human patients with tongue cancer. Unfortunately, we could not detect the L285P mutation in EHF among 15 human patients with tongue cancer, though some 


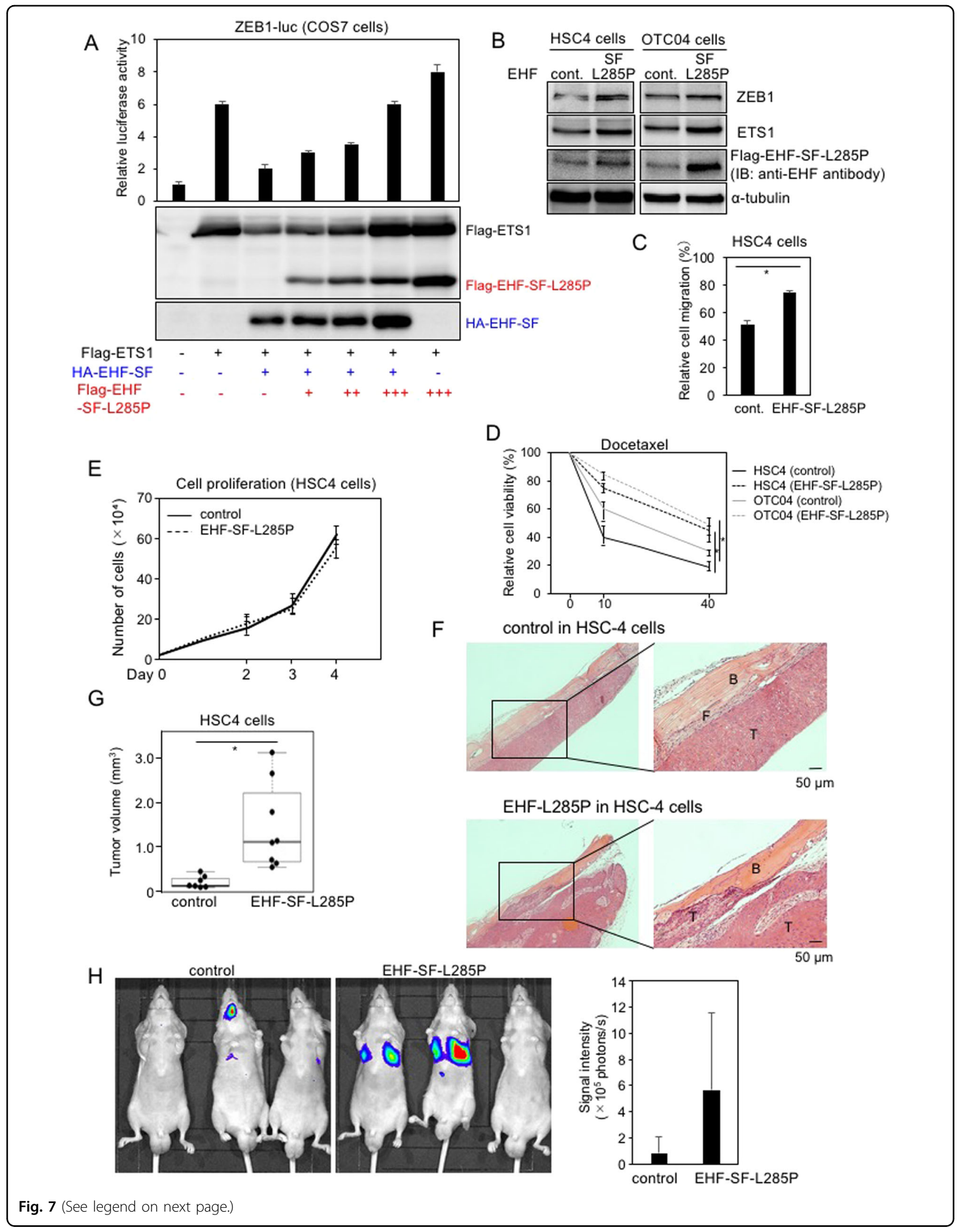


(see figure on previous page)

Fig. 7 Effect of EHF-SF-L285. A ZEB1 promoter activities were determined by luciferase assays (top panel), followed by IB (bottom panel), after cells had been transfected with the indicated plasmids. Each value represents the mean \pm s.d. of triplicate determinations from a representative experiment. Similar results were obtained in at least three independent experiments. B, C, D, E, F, and G HSC4 and OTC04 cells were infected with either control (cont.) or flag-tagged EHF-SF-L285P and subjected to IB (B), motility assays (C), chemoresistance assays (D), and proliferation assays (E). HSC4 cells infected with lentiviruses carrying either control $(n=7)$ or flag-tagged EHF-SF-L285P $(n=8)$ were injected into the periosteal region of the parietal bone in mice. Five weeks later, mice were sacrificed and specimens were prepared after decalcification. Typical histology in hematoxylin and eosin staining are shown $(\mathbf{F})$, followed by statistical analysis $(\mathbf{G})$. T tumor, B bone, F fibrous tissue. $p$ values were determined by Student's $t$-test. ${ }^{*} p<$ 0.01. H Human osteosarcoma 143B-Luc cells infected with either control $(n=5)$ or EHF-SF-L285P $(n=5)$ were injected intravenously into the lateral tail vein of mice. After mice were anesthetized with isoflurane on day 14, D-luciferin potassium salt was injected intravenously, followed by measurement of the emission intensity using the IVIS Lumina imaging system (left) and quantification analysis (right).

mutations were found in the ESE domain (Fig. S5). Especially, an insertion was found at position 288 where a missense mutation (A332P) was detected in ELF3 in ampullary adenocarcinomas. Surprisingly, genomic mutations of ELF3 were frequently found in ampullary adenocarcinomas and other cancers, while they were not detected in tongue cancer. The salivary gland is reported to have the high EHF expression in normal tissues ${ }^{23}$. Therefore, EHF, rather than ELF3, would be very involved in cancer progression in HNSCC.

\section{Materials and methods \\ Cell lines}

Eleven human HNSCC cells (SAS, HSC2, HSC3, HSC4, HOC313, TSU, CA9-22, OSC19, OSC20, OBC01, and OTC04), African green monkey kidney COS7 cells, human breast cancer MDA-MB-231 and MCF7 cells, and human embryonic kidney epithelial HEK293T cells were used in this study. HSC2, HSC3, and HSC4 were kind gifts from Dr. F. Momose and Dr. H. Ichijo ${ }^{24,25}$. CA9-22 was purchased from the Japanese Cancer Research Bioresources (Tokyo, Japan). Other HNSCC cell lines gifts from Dr. E. Yamamoto and Dr. S. Kawashiri as described previously ${ }^{19}$. HEK293T, HeLa, MDA-MB-231, MCF7, and COS7 were obtained from the American Type Culture Collection (Manassas, VA, USA). The cells were authenticated by Single Tandem Repeat analysis and cultured in DMEM (Nacalai tesque, Kyoto, Japan) supplemented with $10 \%$ heat-inactivated FBS, $500 \mathrm{units} / \mathrm{ml}$ penicillin, and $500 \mu \mathrm{g} / \mathrm{ml}$ streptomycin at $37^{\circ} \mathrm{C}$ in a humidified atmosphere containing $5 \% \mathrm{CO}_{2}{ }^{26}$. Cell culture supenatants are tested for mycoplasma contamination using TaKaRa PCR Mycoplasma Detection Set (Takara-Bio, Kusatsu, Japan).

\section{DNA constructs}

The human ZEB1 promoter reporter (hZEB1-Luc) and expression plasmids encoding human ELF3 were previously described $^{10,14}$. cDNAs of ELF5 and EHF-LF were synthesized by a PCR-based strategy using cDNA prepared from breast cancer cells. EHF-SF was constructed by a PCR-based strategy using EHF-LF as a template. Other plasmids used are described elsewhere ${ }^{10}$. Point mutations in EHF were introduced by PCR-based mutagenesis. All constructs were subcloned into the pcDNA3.0 expression vector with a flag tag at the $\mathrm{N}$-terminus and confirmed by sequencing. The primers used for cloning are listed in Table S1.

Additional information on reagents and experimental procedures are described in the Supplementary Materials and Methods.

\begin{abstract}
Acknowledgements
We also thank Mr. T. Kamada, Dr. T. Shirakihara, Dr. N. D. Sinh, Dr. T. Ikeda, and Dr. K. Miyazono for their helpful advice. This work was supported by The Mitsubishi Foundation and JSPS KAKENHI Grant Numbers $15 \mathrm{H} 05018$ and $18 \mathrm{H} 02969$.

\section{Author details}

${ }^{1}$ Department of Biochemistry, Graduate School of Medicine, University of Yamanashi, Yamanashi, Japan. ${ }^{2}$ Department of Otolaryngology, Head and Neck Surgery, Graduate School of Medicine, University of Yamanashi, Yamanashi, Japan. ${ }^{3}$ Center for Medical Education and Sciences, Graduate School of Medicine, University of Yamanashi, Yamanashi, Japan. ${ }^{4}$ Department of Oral Pathology, Graduate School of Medical and Dental Sciences, Tokyo Medical and Dental University, Tokyo, Japan. ${ }^{5}$ Department of Molecular Pathology, Graduate School of Medicine, The University of Tokyo, Tokyo, Japan.

${ }^{6}$ Department of Orthopaedic Surgery, Graduate School of Medicine, University of Yamanashi, Yamanashi, Japan. 'Department of Oral and Maxillofacial Surgery, Graduate School of Medicine, University of Yamanashi, Yamanashi, Japan. ${ }^{8}$ Department of Neurosurgery, Graduate School of Medicine, University of Yamanashi, Yamanashi, Japan. ${ }^{9}$ Department of Health Sciences, Graduate School of Medicine, University of Yamanashi, Yamanashi, Japan. ${ }^{10}$ Department of Pathology, Saitama Medical University International Medical Center, Saitama, Japan
\end{abstract}

Conflict of interest

The authors declare no competing interests.

Publisher's note

Springer Nature remains neutral with regard to jurisdictional claims in published maps and institutional affiliations.

Supplementary information The online version contains supplementary material available at https://doi.org/10.1038/s41389-021-00313-2.

Received: 3 September 2020 Revised: 26 January 2021 Accepted: 11 February 2021

Published online: 12 March 2021 


\section{References}

1. Argiris, A., Karamouzis, M. V., Raben, D. \& Ferris, R. L. Head and neck cancer. Lancet 371, 1695-1709 (2008).

2. Smith, A., Teknos, T. N. \& Pan, Q. Epithelial to mesenchymal transition in head and neck squamous cell carcinoma. Oral. Oncol. 49, 287-292 (2013).

3. Nieto, M. A. Context-specific roles of EMT programmes in cancer cell dissemination. Nat. Cell Biol. 19, 416-418 (2017).

4. Pastushenko, I. \& Blanpain, C. EMT transition states during tumor progression and metastasis. Trends Cell Biol. 29, 212-226 (2019).

5. Pastushenko, I. et al. Identification of the tumour transition states occurring during EMT. Nature 556, 463-468 (2018).

6. Shibue, T. \& Weinberg, R. A. E. M. T. CSCs, and drug resistance: the mechanistic link and clinical implications. Nat. Rev. Clin. Oncol. 14, 611-629 (2017).

7. Saitoh, M. Involvement of partial EMT in cancer progression. J. Biochem. 164 257-264 (2018).

8. Horiguchi, K. et al. TGF- $\beta$ drives epithelial-mesenchymal transition

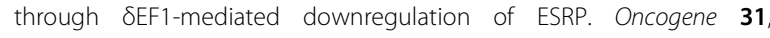
3190-3201 (2012).

9. Garrett-Sinha, L. A. Review of Ets1 structure, function, and roles in immunity. Cell Mol. Life Sci. 70, 3375-3390 (2013).

10. Sinh, N. D., Endo, K. \& Miyazawa, K. Saitoh M. Ets1 and ESE1 reciprocally regulate expression of ZEB1/ZEB2, dependent on ERK1/2 activity, in breast cancer cells. Cancer Sci. 108, 952-960 (2017).

11. Luk, I. Y., Reehorst, C. M. \& Mariadason, J. M. ELF3, ELF5, EHF and SPDEF transcription factors in tissue homeostasis and cancer. Molecules 23, 2191, https://doi.org/10.3390/molecules23092191 (2018).

12. Yachida, S. et al. Genomic sequencing identifies ELF3 as a driver of ampullary carcinoma. Cancer Cell 29, 229-240 (2016).

13. Fukagawa, A., Ishii, H., Miyazawa, K. \& Saitoh, M. SEF1 associates with DNMT1 and maintains DNA methylation of the E-cadherin promoter in breast cancer cells. Cancer Med. 4, 125-135 (2015).
14. Shirakihara, T., Saitoh, M. \& Miyazono, K. Differential regulation of epithelial and mesenchymal markers by $\delta$ EF1 proteins in epithelial mesenchymal transition induced by TGF- $\beta$. Mol. Biol. Cell 18, 3533-3544 (2007).

15. Neve, R. M. et al. A collection of breast cancer cell lines for the study of functionally distinct cancer subtypes. Cancer Cell 10, 515-527 (2006).

16. Kutay, U. \& Guttinger, S. Leucine-rich nuclear-export signals: born to be weak. Trends Cell Biol. 15, 121-124 (2005)

17. Jans, D. A., Martin, A. J. \& Wagstaff, K. M. Inhibitors of nuclear transport. Curr. Opin. Cell Biol. 58, 50-60 (2019).

18. Kim, R. et al. The effect of induction chemotherapy using docetaxel, cisplatin, and fluorouracil on survival in locally advanced head and neck squamous cell carcinoma: a meta-analysis. Cancer Res. Treat. 48, 907-916 (2016).

19. Nakamura, R. et al. Transforming growth factor- $\beta$ synthesized by stromal cells and cancer cells participates in bone resorption induced by oral squamous cell carcinoma. Biochem. Biophys. Res. Commun. 458, 777-782 (2015).

20. Dittmer, J. The role of the transcription factor Ets 1 in carcinoma. Semin. Cancer Biol. 35, 20-38 (2015).

21. Lu, G. et al. Phosphorylation of ETS1 by Src family kinases prevents its recognition by the COP1 tumor suppressor. Cancer Cell 26, 222-234 (2014).

22. Nishida, T., Terashima, M. \& Fukami, K. PIASy-mediated repression of the Ets-1 is independent of its sumoylation. Biochem. Biophys. Res. Commun. 345, 1536-1546 (2006).

23. Kleinbaum, L. A. et al. Human chromosomal localization, tissue/tumor expression, and regulatory function of the ets family gene EHF. Biochem. Biophys. Res. Commun. 264, 119-126 (1999).

24. Ichijo, H., Momose, F. \& Miyazono, K. Biological effects and binding properties of transforming growth factor- $\beta$ on human oral squamous cell carcinoma cells. Exp. Cell Res. 187, 263-269 (1990).

25. Momose, F. et al. Variant sublines with different metastatic potentials selected in nude mice from human oral squamous cell carcinomas. J. Oral. Pathol. Med. 18, 391-395 (1989).

26. Shirakihara, T. et al. TGF- $\beta$ regulates isoform switching of FGF receptors and epithelial-mesenchymal transition. EMBO J. 30, 783-795 (2011). 